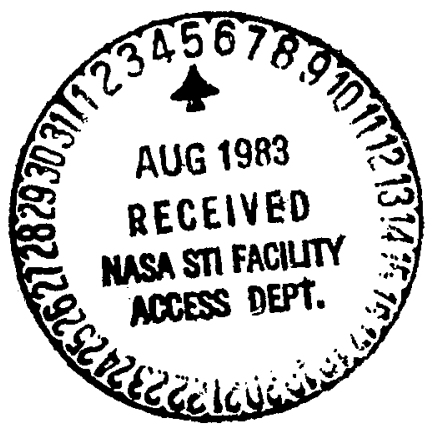

\title{
The Evaluation of the Rolling Moments Induced by Wraparound Fins
}

Arnan Seginer and Benjamin Bar-Haim

(NASA-TM-84381) THE EVALUATION OF THE

N $83-29180$

ROLLING MOAENTS INDUCED BY WRAPAROUND $r^{\prime} L N S$

(NASA) $13 \mathrm{p}$ HC AO2, MF AO1 CSCL O IA

$63 / 02 \quad 28310$

June 198

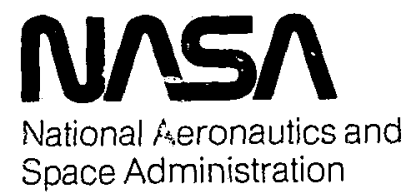




\section{The Evaluation of the Rolling Moments Induced by Wraparound Fins}

Arnan Seginer, Ames Research Center, Moffett Field, California

Benjamin Bar-Haim, Technion-Israel Institute of Technology, Haifa, Israel

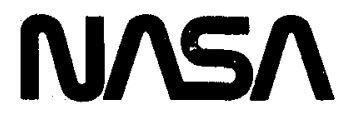

National Aeronautics and

Space Administration

Ames Research Center

Moffett Field, California 94035 


\author{
Arnan Seginer* \\ NASA Ares Research Center, Moffett Field, California \\ and \\ Benjamin Bar-Haim ${ }^{\dagger}$ \\ Technion-Israel Institute of Technology, Haifa, Israel
}

\section{Abstract}

A possible reason is suggested for the induced rolling moments occurring on wraparound-fin configurations in subsonic flight at zero angle of attack. The subsonic potential flow over the configuration at zero incidence is solved numerically. The body is simulated by a distribution of sources along its axis, and the fins are described by $A$ yortexlattice method. It is shown that in ing moments can be induced on the antisymmetric $1 \%$. by the radial flow generated at the base of the configuration, either over the converging separated wake, or over the diverging plume of a rocket motor.

\section{Nomenclature}

$\mathrm{C}_{\ell}=$ rolling-moment coefficient, normalized by body diameter and body cross-section area

$\mathrm{C}_{\ell_{\mathrm{p}}} \quad=$ roll-damping-moment coefficient, sec/rad

$C_{\ell_{\delta}} \quad=$ rolling-moment coefficient slope due to fin deflection, $1 /$ deg

$\mathrm{C}_{\mathrm{p}} \quad=$ pressure coefficient

$d \vec{R}=$ line-vortex segment vector

$d \hat{V}=$ velocity induced by line vortex segment, Eq. (8)

D $\quad$ = body diameter

[H] = influence coefficient matrix, Eq. (9)

I = number of souxce or vortex and column number in the matrix of Eqs. (7) and (9)

I $\quad=$ number of collocation point and line number in Eqs. (7) and (9)

$\mathrm{M}_{\mathrm{S}} \quad=$ total number of sources used to describe the body

$M_{\infty} \quad=$ free-stream Mach number

n $\quad=$ unit vector normal to aerodynamic surfaces

$\mathrm{N}_{\mathrm{v}} \quad=$ total number of fin panels

$\mathrm{P}_{j} / \mathrm{P}_{\mathrm{co}}=$ rocket motor pressure ratio

*National Research Council - Research Associate, Aerodynamics Research Branch; Associate Professor, Technion-IIT. Member AIAA.

Traduate Student, Department of Aeronautical Engineering.

$$
\begin{aligned}
& \text { \{Q\} = source-intensities vector } \\
& \vec{r} \quad=\text { radius vector, } \mathrm{Eq} \cdot(8) \\
& r_{B} \quad=10 c a l \text { body radius } \\
& \left(u_{x}, u_{x}\right)=\text { velocities in system }(x, r, \theta) \\
& \left\{\mathrm{U}_{\mathrm{n}}\right\}=\text { vector of normal velocities due to the }
\end{aligned}
$$

\section{Introduction}

The last decade has seen the development of many tube-launched missiles, from ground-launched, saturation field-artillery rockets to air-

launched weapons, and from simple unguided rockets to sophisticated cruise missiles. Tube launching was chosen for its packaging convenience and to increase the reliability of the rocket motor. However, such a launching procedure requires folding aerodynamic stabilizers because of its packaging constraints. The stabilizers would be folded 


\section{OWIGTSE PREE TE \\ OF POOR QUALTY}

in the stowed position to fit within a circular cylinder and would deploy instantly after launch. Wraparound-fin (WAF) configurations (see Fig. 1) meet this requirement and at the same time maximize the volume available for the missiles subsystems, especially for the nozzle exit.

It has been repeatedly observed that WAF configurations have conventional Iongltudinal aerodynamic characteristics ${ }^{1-4}$ equel to those of configurations with planar fins of Identical planform. However, as early as 1960 , Featherstone ${ }^{1}$ pointed out that WAF configurations displayed a seemingly unpredictable lateral behavior. Of special interest for the present investigation were the induced rolling moments. While these moments were understandable (if not predictable) at the higher angles of attack as a result of the asymmetry of the curved fins, ${ }^{4,5}$ the moments measured at zero angle of attack ${ }^{5}$ presented an enigma. An even greater enigma was the rull reversal that occurred when WAF configurations accelerated or decelerated through the sonic-speed range. 5

Because of the significance of WAF configurations in weapons technology and their unusual lateral characteristics, an international research program [the Technical Cooperation Program (TTCP) ${ }^{7}$ ] was set up in 1969 at a meeting at Eglin AFB. In the ensuing research effort (encompassing the U.S. Armed Forces, the United Kingdom, Canada, and Australia) an intensive experimental investigation program was launched to study the aerodynamic characteristics of a standard configuration (FIg. 1) with 20 different fins and two different bodies, at Mach numbers from 0.3 to 3.0 (Refs, 8-10).

The experimental results of this program proved that the static longitudinal aerodynamic characteristics of WAF configurations did not differ from those of conventional configurations with identical bodies and flat fins, if the flat fin area was equal to the projected area of the curved fins. This conclusion meant that WAF configurations could be used where flat fins had been used before, without any penalty in their longitudinal aerodynamics and even without a severe increase in their total drag. However, those results also substantiated Featherstone' $s^{1}$ findings of unusual lateral aerodynamics. In spite of the relatively large scatter in the experimental data obtained from the stingmounted models in the various wind tunnels involved, 10 there was no doubt that WAF configurations developed induced rolling monents, even at zero angle of attack. Generally speaking, these misments acted upon the fins in the direction of tivir center of curvature at subsonic speeds and changed direction in transition to supersonic speeds, or vice versa. This behavior could be detrimental to the stability of WAF missiles because roll resonance, or pitch-rold lock-in and catastrophic yaw, due to roll-yaw coupling could develop at the point of roll reversal, increasing the scater of such missiles around their targets.? Mlthough the problem can be overcome by intentionally spinning the missile (by means of canted fins, for example) the TTCP participants still felt the need to identify the origin and to understand the nature of the induced rolling moments. However, In spite of years of investigative effort, the answer still eluded them.

Intrigued by this problem, the authors tried an analytic approach. A lifting-line theory for antisymetric wings with spanwise curvature in uniform flow was developed, with the intent that the asymetric tip vortices generated at any finite angle of attack would retain some vorticity when the angle was gradually decreased to zero. However, nothing but the trivial solution could be found at zero incidence. Next, the authnrs modified the vortex-lattice cocie for wings alone ${ }^{11}$ to handle antisymmetric, spanwise-curved wings. Agaịn, wraparound fins at zero incidence in uniform flow failed to produce rolling moments. With the failure of both these approaches in uniform oncoming flow, and with some experimental indication ${ }^{6}$ that rocket-motor burnout also gave rise to roll reversal, the authors suspected that, in subsonic flow, the rolling moments could be generated by a radial-velocity component induced on the fins by the wake flow field.

The purpose of this work was to investigate this idea and to prove its feasibility. It was not meant to be a rigorous solution of the flow field around a WAF configuration at any given angle of attack. Consequently, the authors decided to use existing approximate numerical methods with some simplifying assumptions in order to obtain at least a qualitative answer at a minimum effort and cost.

\section{The Mathematical Mode1}

The mathematical model, as well as the numerical methods used here, is well. known and is described only briefly. The interested reader may consult the relevant references for more details.

Since there is no reason to expect viscous effects in the mechanism generating the rolling moments, it is sufficient to describe the potential flow about the WAF configuration. If the filow is incompressible it can be described by the wellknown Laplace equation for the velocity potenti. 1

$$
\nabla^{2} \phi=0
$$

where $\phi$ is the potential function, and by the tangency boundary condition on all solid surfaces of the configuration:

$$
\frac{\partial \phi}{\partial n} \cdot \vec{n}=0
$$

where $\vec{n}$ is the unit vector normal to the surface. Further boundary conditions can be imposed such as the Kutta condition at the fins' trailing edge, and/or a prescribed wake geometry (1inear wake), or a force-free, rolled-up wake (nonlinear wake). Such a problem can be solved in a given uniform flow by an assumed distribution of a discrete number of potential elemental functions (i.e., sources, doublets, and vortices) inside the body or on $i t s$ surfaces. The intensities of these functiuns are determined by satisfying the boundary conditions. With the intensities known, the induced perturbation velocities at every point in the flow field and the pressure distribution over the configuration's surfaces can then be computed.

If the solution has to be extended into the subsonic compresstble flow regine, as is done in this work, then the linearized, small-perturbation potential equation 


$$
\left(1-M_{\omega}^{2}\right) \phi_{x^{\prime} x^{\prime}}^{\prime}+\phi_{y^{\prime} y^{\prime}}^{\prime}+\phi_{z^{\prime} z^{\prime}}^{\prime}=0
$$

that approximntuly describes the compressible flow is transformed into the Incompressible potential Eq. (1.) by the Gothert transformation:

$$
x=x^{\prime} / \beta, \quad y^{x}=y^{\prime}, \quad z=z^{\prime}
$$

where the primed quantities belong to the physteal compressible flow and the unprimed ones to the cransformed incompressible flow, and the same solution procedure, described above, is used. With this approach, what remains to be decided is the exact model of the potential singular-element distribution that is to be used in a certain case.

As stated before, the purpose of this work was to identify (even if only qualitatively) a possible mechanism that could induce rolling moments on WAF configurations at zero angles of attack. It was therefore dectded to use the stmplest and least expensive available numerical approach. Consequently, the subsontc flow field ove: WAF configurations was solved at zero angle of attack only. 'this restriction grencly simplified the modeling of the body and saved computation time. It did not soverely linit the applifability of the calculated resules since most WAF missiles fly at rather small angles of attack and the rol.jing moments at these angles are practicitly constant. ${ }^{a}$ It was assumed, and later justlefed by the computed results, that at zero angle of attack the solution could be uncoupled. Since the fins were not generating any lift (except for the minute amount necessary for the induced roliting moment), their effect on the flow fluld, and consequently on the solution for the body, is belfeved to be negligtble. Tt was cherefore decided to stmulate the body only first by a distribution of sources along its axis, and then to simulate the fins in the presence of the body by a modification of the nonilnear vortex1atitice method of Ref. 11. This uncoupled solution minimized the computation time, because the bodyindueed flow field had to be computed only once, and then to could be used for all subsequent fin at rangements.

\section{Body Modeling}

A good approxtmation of the body-induced flow lield could have been obcaned using one of the now-classic: sunrce-pincl methods (such as discussed in Rof. 12). An even betcer approximation could have been whtained by the simultaneous solucton for the cottplete suteraction flow fleld of the body and the whaparound surfaces with the method of Raf. 13. This method al so would have accounted for the clleets of the lins and thetr wakes on the body at Fintle angles of attack. llowever, such mothods ate time-consuming and unjustifled for a qualitative Investignation such as this and, as later ubserved, not necessary at zero angle of attack.

In this work the actual description of the axisymunecte body at zero incidence is by a distribution of a fintte number of discrete point sources along its axis. The intensities of these sources are determined by satisfyding the flowtangensy boundary condition [Eq. (2)] on the same number of collocation potnts on the body. The details of this well-established method are given by von karmon ${ }^{14}$ and are only summartzed here.
OFTENAE PAGE IS
OF POOR QUALITY

Let $\{Q(I)\}$ be the vector of the source Intensitfes, and let $\left\{x_{c}(I)\right\}$ designate the locations along the body axis of these sources. The velocity at any given point $(x, r)$ in the cylindrical system $(x, r, 0)$, Induced by the sources system, is given by

$$
\left.\begin{array}{l}
u_{x}(x, r)=\sum_{I=1}^{M_{s}} Q(I) \frac{x-x_{c}(I)}{\left\{\left[x-x_{c}(I)\right]^{2}+r^{2}\right\}^{3 / 2}} \\
u_{r}(x, r)=\sum_{I=1}^{M_{S}} Q(I) \frac{r}{\left\{\left[x-x_{c}(I)\right]^{2}+r^{2}\right\}^{3 / 2}}
\end{array}\right\}
$$

where $M$ is the total number of sources required to describe the body. The flow-tangency condition [Eq. (2)] on the body surface is

$$
\frac{d r_{1}}{d x}=\frac{u_{r}}{U_{\infty}+u_{x}}
$$

Equations (5) and (6) combine at any one of the collocation polnts $\left\{x_{p}(J)\right\}$ on the body surface lnto the following matrix cquacion:

$$
\begin{gathered}
{\left[\frac{x_{B}(J)-\left[d r_{B}(J) / d x\right]\left[x_{1}(J)-x_{c}(I)\right]}{\left[\left[x_{p}(J)-x_{c}(I)\right]^{2}+r_{B}(J)^{2}\right]^{3 / 2}}\right]\{Q(I)]} \\
\quad\left\{\left\{l_{\infty} \frac{d r_{B}}{d x}(J)\right\} \quad J, I=1,2, \ldots, M_{S}\right.
\end{gathered}
$$

Satisfuction of the boundary condition [Bq. (7)] at one of the control points may be replaced by an imposed stagnation condtion at the body apex to improve the accuracy of the solution in this region. ${ }^{25}$ The linear system Eq. (7) is solved by the Gauss elimination method for the source intensities $\{Q(I)\}$. With the intensities of the sources known, the velocittes induced by the body can bo calculated at any polnt in the flow fleld using Eqs. (5).

Obviously, the deseription of the body by a source distribution along ths axis is valid for an axisymmetric body at zero incidence only. For any othev case, a more sophisticated scheme (such as that of Ref. 1.2) must be used; however, this description sufficed for the needs of this investigation. Furthermore, the source distribution was computed unly once in the absence of the fins (In order to save computation tine) by assumlng that the Interaction of the fins with the body wis negligible. This interaction was evaluated separately from the results of the complete program, and the assumption was verified when it was found that the velocities induced by the fins on the body nt aero inctelence were negligible. 'lhis was expected because, at this condtiton, the fins do not generate lift and therefore do not disturb the Elow.

\section{Wake Modeling}

As mentioned beforc, when simulation of wraparound surfaces in uniform flow falled to produce rolling moments, the authors dedueed from the rollt reversal. at sonfe speeds and from the opposite sense of the roliling moment when the WAF-uissile's rocket motor was firing, that the wake structure must have nu upstream influence (in subsonte flow) on the fins. $\Lambda$ radial veloctty component induced 
on the antisymmetric fins could generate an asymmetric load on them and, therefore, would also generate a rolling moment.

With this in mind, it became necessary to simulate not only the body, but also its wake. Since the potential flow model used could not account for a wake (neither the viscous wake of the body nor the plume of a rocket motor), the problem coul.d be evaded by substituting a solid body for the inner wake enclosed by the dividing streamline. For this to be done, the shape of the dividing streamline had to be known from external sources, and the resulting "solid body" was incorporated into the body simulation as if it were its inherent rear part.

Three types of wake shapes are simulated in this work: 1) the free-undisturbed wake of a body in free flight; 2) the wake of a sting-mounted body in a wind tunnel; and 3 ) the plume of an underexpanded jet firing from a rocket-motor exit nozzle. Wake shapes are not readily available in any single reference, and a comprehensive literature review had to be unciertaken in order to compile the necessary data. ${ }^{16}$ In view of the length of the present paper, only the relevant conclusions of this review are given.

1) The dividing streamline of a free wake was modeled by an ogival solid body. This shape was based on the experimental observations in Refs. 17 through 20. The flow visualization discussed in Refs. 17, 18, and 19 showed the separating streamline to leave the body tangentially to the surface, and to enter the rear stagnation point at an angle between $30^{\circ}$ and $40^{\circ}$. The tangent ogive used in the present model offered a good simulation of this pattern (Fig. 2). The most important characteristic of the wake - namely, its length to the rear stagnation point and its dependence on the Mach number (Fig. 2) - was based on Ref. 18. The Reynolds number did not affect the length and shape of the wake once the wake was turbulent.18,20 The results of the experiments in Ref. 20 with different base shapes indicated that the presence of the fins should have no effect on the shape and total length of the wake. When the computer code was run, the influence of variations in the wake shape were investigated. Various wake shapes (e.g., parabolic or cubic polynomial) of equal length were tried, but the effects of these variations on the results were insignificant. 16

2) Verification of the proposed mathematical model required a comparison with experimental data. However, such data were obtained from stingmounted, wind-tunnel models. The sting changed the wake into that of a backward-facing step, varying from an axisymmetric step ${ }^{19}$ (large step-heightto-body-diameter ratio) to an almost two-dimensional step ${ }^{17}$ (when the step is sma11). The location of the rear stagnation point (or reattachment point) on the sting has not been previously reported. The authors determined its approximate position from mensured pressure distributions ${ }^{21}$ and flow visualizations, 19 and defined an equivalent ogival afterbody by an extrapolated rear stagnation point on the axis (Fig. 2). The dependence of the length of this equivalent wake on the flow Mach number is shown in Fig. 2 for a body-to-sting-diameter ratio of $3: 1$. This ratio was chosen because it is widely used by wind-tunnel operators, and because it was used in the Arnold Engineering and Development
Center (AEDC) 4-Ft Wind Tunnel subsonic and transonic experiments reported by Dahlke. 9 Although the method of determining the position of the rear stagnation point is fairly crude, the influence of its inaccuracy is not large. The change in the induced rolling moment caused by a $20 \%$ variation in the length of the wake was in the range of $1-2 \%$.

3) The third wake simulated was a rocketexhaust jet plume. WAF configurations are rocketpowered, and the induced rolling moments during the powered phase of the flight are also of interest. ${ }^{6}$ The literature review of Ref. 16 defined a large number of parameters that determine the shape of the plume. Since the prime objective of this work was to obtain qualitative data on the induced rolling moments, and since experimental data on these moments during powered flight were not available for comparison, the plume was modeled for a limited number of parameters only. These parameters were for a sonic jet, blowing from a cylindrical nozzle exit into a subsonic outer flow. The jets were underexpanded, with jet-to-free-stream pressure ratios from 1 through 6 . The typical geometrical features of the jet plume (the length of the first jet "diamond," its upstream vertex angle, and the dialleter of its downstream end) under these conditions wure taken from Refs. 22 and 23 . With these three parameters specified, the shape of the solid body simulating the jet plume was modeled by a second-order polynomial. 16

\section{Fin Modeling}

With the body-induced flow field known, the pressure loading on the fins can be calculated and integrated to produce the induced rolling moment. In the present work, this is done by a nonlinear vortex-lattice method (VLM). 11 Each fin is divided into quadrilateral panels (Fig. 3), and bound horseshoe vortices of unknown strength are distributed at the quarter chord positions of the panels (inset A in Fig. 3). The trailing vortices from the system are free to shed from the fins' trailing and side edges, and after determining their equilibrium position in space, they form the vortex wake of the fins. The intensities of the vortices are again determined by the tangencyboundary condition [Eq. (2)]. This condition is imposed at a finite number of control points located at the midspan of each panel's $3 / 4$ chord line. This tangency condition is satisfied by the mutual cancellation of the velocity components due to the free stream, body, and vortex flow fields that are normal to the fins' surfaces at the control points.

The velocity $d \vec{v}$, induced by a segment $\vec{d} \vec{l}$ of a line vortex of strength $\gamma$ at position $\vec{r}$, is given by the Biot-Savart Law:

$$
d \vec{V}=\frac{\gamma}{4 \pi} \frac{d \vec{l} \times \vec{I}}{r^{3}}
$$

When the contributions of all the vortices are integrated, the total induced velocity at any given point can be calculated. The component $V_{n}$ of the total induced velocity that is normal to the fin's surface at a set of control points located at each panel's $3 / 4$ chord is given by

$\nabla_{n}(J)=\sum_{I=1}^{N_{v}} \mathrm{H}(J, I) \gamma(I) \quad J=1,2, \ldots, N_{v}$ 
where $\mathrm{N}_{\mathrm{v}}$ is the total number of vortex panels with strengths $\gamma(I)$, and the matrix [H] is the influence coefficient matrix of all vortices (I) on points (J). An influence coefficient is defined as the component, normal to panel (J) at its control point, of the velocity induced there by a vortex of unit strength at (I). The tangency boundary condition at the control points is satisfied when the velocity component normal to the surface (that is, induced by the vortices) is cancelled by the normal component of the combined uniform and body-induced flow fields

$$
\left\{U_{n}\right\}+[H]\{\gamma\}=0
$$

Satisfying this tangency boundary condition [Eq. (10)] at a given number of control points results in the same number of linear algebraic equations for the vector of the unknown intensities of the vortices $\{\gamma\}$. The solution of this system of equations requires, however, prior knowledge of the trajectories of the free vortices in order to account for the velocities induced by them on the control points. The trajectories, in themselves part of the solution, are either assumed to be known in the Iinear VLM codes or computed by an iterative process in the nonlinear VLM codes. ${ }^{13}$ In the latter case, an initial guess for the trajectories is needed. It is usually assumed that the free vortices trail off to infinity as straight lines. With the intensities of the vortices known, the free vortices are allowed to follow local streamlines. The vortex intensities are then recomputed and the process is reiterated until the vortex wake relaxes to its equilibrium rolled-up position. This nonlinear procedure was also followed in the present investigation. ${ }^{16}$ However, it was found that since the fins were at zero incidence and the normal forces generated by them were of second order, their influence on the wake roll-up was almost insignificant. Consequently, the contribution of the nonlinear process to the induced rolling moments was negligible, and they could be computed by the linear version of the code (its first iteration only) at a considerable reduction (75\%) in computation time.

With the strengths of the vortices known, the pressure distribution acting on the fins is computed by the Kutta-Joukowski theorem and is integrated relative to the body axis to give the rolling moment.

\section{Computational Results}

The main purpose of this work was to identify a possible mechanism inducing rolling moments on WAF configurations at zero angle of attack. The preliminary study indicated that, while no such moments could be produced in a uniform flow field, the radial velocity component at the base of the configuration (inward over a closed wake or outward over a jet plume) could induce rolling moments by interacting with the antisymetric fins.

To verify this hypothesis (even if only qualitatively), the computation results had to be compared with experimental data. No such data were available for WAF configurations in free flight so wind-tunnel data generated by the TTCP (Ref. 7) had to be used. This was the reason for the simulation of the wake of a sting-mounted mode1. There were, however, drawbacks to the use of these data. Not having been aware of the posstbly decisive role of the wake flow in this case, the participants of TTCP did not specify in their reports (e.g., Refs. 8-10) the model-to-sting-diameter ratios. One cannot be certain that the same ratio was used throughout the program. As shown in Fig. 2 this ratio has a first-order influence on the length of the wake and consequently also should have a nonnegligible effect on the radial component of the velocity and on the rolling moments it induces. Since the experiments reported in Ref. 9 were done with a body-to-sting-diameter ratio of $3: 1$ (which is the common practice in wind-tunnel work), and because of lack of information to the contrary, it was assumed here that all the data were obtained using this ratio. This assumption may have contributed to the scatter in the data, which already was considerable because of the difficulty in accurately measuring the relativeiy small rolling moment, especially at the lower subsonic Mach numbers. ${ }^{9}$ The low accuracy of the experimental data limited the comparison with the computational results more to qualitative evaluation than to full verification of the mathematical model.

\section{The Standard Mode1}

The TTCP standard WAF configuration (Fig. 1) designated as BIFI in the literature was modeled for the comparison. The configuration was composed of an axisymmetric body (a 2-diam-long ogive nose and an eight-caliber cylindrical body) and a cruciform of fully deployed wraparound fins of rectangular planform that completely encircle the body. when folded (Figs. 1 and 3 ).

A distribution of a minimum of 50 sources was necessary for a reasonably smooth modeling of the body and its wake. The accuracy of the body modeling was controlled by a comparison of the resulting body radius (of the zero streamline) between control points with the actual radius, and by a comparison of the local computed flow direction at these points with the body slope. The number of the sources and their spacing along the axis were automatically adjusted until prescribed accuracy criteria were satisfied.

The modeling of the fins was done with 100 uniform vortex panels (Fig. 3) per fin. This fine division guaranteed the numerical accuracy of the results. For instance, an increalse of the number of panels by $30 \%$ changed the computed rolling moment by less than $0.5 \%$. The computed pressure distribution over a fin that is shown in Fig. 4 indicates that the number of panels could be decreased and computation time could be saved by a nonuniform spacing of the panels with a far lower density on the upstream three-quarters of the fin and a denser division on its aft quartar where the pressure gradients are large. Another method to save computation time at a penalty of a $2-3 \%$ error in the rolling moment was to model onl.y one pair of fins on the configuration and multiply the computed moment by two. A linear solution for one pair of fins took about $125 \mathrm{sec}$ on an IBM $370 / 165$ computer; a cruciform fin configuration required about $500 \mathrm{sec}$.

The linear solution needed a prescribed shape of the fins' vortex wake. Inset B in Fig. 3 describes the options for this shape. The first assumption is that the free vortices lie on a truncated conical surface trailing the fin. The 


\section{ORTGMAR RMEE TS \\ OF POOR QUALITY}

user can specify the length and vertex angle of this cone and even a spiral twist of the vortices over the cone as a simulation of the wake roll-up. A nonlinear computation of the full-wake roll-up (found to be unnecessary for the present purpose) would require considerably more computer time.

Comparison with Experimental Data

In Fig. 5 the variation of the rolling-moment coefficient for the standard WAF configuration with the Mach number is compared with the experimental data of Ref. 10. These data were obtained with the fins in a cruciform ( $t$ ) position and in an $(x)$ position, and also with special miniature fin balances in addition to the main integral balance. The agreement between the computed results and the experimental data is good at Mach numbers 0.5 and 0.8 . The discrepancy at $M=0.3$ cannot be explained by the present model. The general trend of the declining rolling moment with increasing Mach number is correctly obtained. The Goethert compressibility correction cannot be carried past $M=0.8$, and the curve is extrapolated (dashed line) to the vanishing value measured at $M=1.0$. Although not computable, this vanishing of the rolling moment at sonic speeds can be qualitatively explained by the present mathematical model. As the flow over the base of the body turns supersonic, the turning of the flow (either inward or outward in the wake region) is centered on the base corner, and cannot exert any influence upstream on the fins. Thus, the fins experience only parallel flow and, consequently, no rolling moment is developed. Comparisons with wind-tunnel results of other WAF configurations are given in Ref. 16 and show the same kind of agreement as is shown in Fig. 5. Also shown in Fig. 5 for comparison purposes is the rolling-moment coefficient for the same configuration in free flight. The shorter and more rapidly closing wake in free flight almost doubles the computed rolling moments. These results could not be verified experimentally for lack of such data.

Other comparisons that were possible with experimental data are shown in Figs. 6 through 8 . Figure 6 compares the rolling-moment cocfficients at $M=0.8$ for the standard configuration with the fins at different opening angies $\left(\theta_{D}\right)$, where at $\theta_{D}=120^{\circ}$ the fins are completely folded, and at $\theta_{D}=0^{\circ}$ they are fully deployed. Again, good agreement is observed, except for opening angles of $25^{\circ}$ and $45^{\circ}$. No explanation can be offered for the discrepancy at these two angles. The same code was also used to compute the rolling moments generated by a small antisymmetrical deflection of the fins at an angle $(\delta)$. The resultes are linear (Fig. 7). The computed slope of the curve, $\left(C_{\ell}\right)_{\delta}=0.131 / \mathrm{deg}$, falls close to the experimental value of $\left(C_{\ell}\right)_{\delta}=0.138 / \mathrm{deg}$. The final comparison was of the computed roll-damping coefficient $\left(C_{\ell_{p}}\right)$ with experimental data. To compute this parameter, the whole flow field was spun around the configuration with a velocity distribution adopted from two concentric cylinders with the outer cylinder at infinity. The results are compared in Fig. 8 with data from the AEDC and Jet Propulsion Laboratory (JPL) experiments reported in Ref. 9. The dashed line describes the results of the present mathematical model, and the solid line is obtained from these results by adding the roll damping due to the body boundary layer. The results are in fair agreement with the AEDC data (circles). Comparison with the JPL data is inconclusive because of the large uncertainty levels reported for these data (the AEDC data had no uncertainty levels shown). 9

\section{Parametric Study}

Several other parameters were investigated, with details shown in Ref. 16. Some examples follow.

Since the main assumption was that the base flow was responsible for the induced rolling moments, aoving the fins upstream should weaken this moment as was demonstrated in Fig. 9. Rolling moments for fins flush with the base flane are compared with those for fins moved upstream half a body diameter and one body diameter, respectiveiy. The rolling moment is clearly decreasing with increasing distance from the base, and extrapolation seems to indicate that it might vanish at some upstream distance of one and one-half to two body diameters from the base. It is interesting to note that tree wraparound fins of Vought's MLRS missile are positioned upstream of the base $^{24}$; however, no explanation was given for this design.

The strong effect of the proximity to the base is demonstrated also in Fig. 10, where the influence of fin leading-edge and trailing-edge sweep angle was studied. While leading edge sweepback reduces the rolling moment only slightly, an identical forward sweep of the trailing edge sharply reduces the rolling moment. This result could be anticipated because most of the flow turning induced by the base flow would be felt by the aft portion of the fin as was indeed demonstrated by the pressure distribution in Fig. 4. Moreover, most of the load there is carried by the outer aft corner of the fin that is sliced off when the trailing edge is swept forward. While there are no experimental data available for swept-forward trailing edges, Ref. 9 reports results for swept-back leading edges. However, the extremely large scatter in these data prevents any significant comparison. A possible experimental. indication of the influence of the wake can be found in the strong effects that changes in the geometry of the fin trailing edge had on the rolling moment. 25

The effect of the span of the fins is shown in Fig. 11. In certain missile designs the span of the fins is increased by increasing the sector angles $(\Psi)$ covered by the fins and by making them overlap (an arc angle of $90^{\circ}$ is the common fin full span). As expected, the larger area of the fin generates a higher rolling moment, but the increase is not linear.

A further demonstration of the strong effect of the wake flow on the fins is presented in the rolling moments due to a jet plume of pressure ratio $P_{j} / P_{\infty}=6$ (Fig. 12). The results are shown for the only case of jet plume that was simulated (see the section on wake simulation) at the highest pressure ratio, because the rolling moment is then at its peak. For lower pressure ratios, the moment decreases in size and vanishes for an ideally expanded jet. As shown in Fig. 12, the outward radial component of the wake flow changes 


\section{ORIGINAL PAGE HS \\ OF POOR QUALITY}

the direction of the rolling moment, which is now about four times as great as the moment induced by the inward flow of the closed wake.

For additional examples (e.g., canard WAF, other asymetric fin configurations, swept fins, etc.) see Ret. 16 .

\section{Conclustions}

By using a source-distribution simulation for the body and a vortex-lattice simulation for the wraparound fins, it is shown that the radial velocity component induced on the fins by the wake flow field could be responstble for the rolling moments encountered by WAF configurations in subsonic flow, at zero angle of attack. No other mechanisms were found; however, their existence cannot be ruled out. The present simulation estimates rolling moments of the right magnitude and correct trends.

Assuming that the mechanism described by the present mathematical model is valid, then the induced rolling moments could be eliminated by mounting the fins about two body diameters upstream of the base of the body. Another method to prevent roll reversal in the accelerating phase is to accelerate into supersonic speed before the rocket motor burns out, because the roll direction is the same in powered subsonic flight and in supersonic flight. "This, however, will not solve the problem when the WAF configuration slows down to subsonic speeds at the end of its filight. The negative rolling moment experienced by WAF configurations in supersonic flow cannot be explained by the present purely subsonic mathematical model. It may be due to shock wave focusing on the concave side of the curved fin with the result tant pressure difference acting in the direction of the convex surface. This hypothesis requires further theoretical work as does a parametric study of various effects on the rolling moments. The authors also intend to apply the source-paned method ${ }^{3}$ to describe the body and the nonlinear vortex-latice method for the fins in order to compute WAF aerodynamics at higher angles of attack.

\section{Acknowledgment}

The work described here was done in partial rulfilinent of the requirements for the M.Sc. degree of B. Bar-Haim.

\section{References}

${ }^{2}$ Featherstone, H. A., "The Aerodynamic Characceristics of Curved Tail Fin," Convair/Pomona Division of General Dynamics Corp., Pomona, Calif., GIDC-ERR-PO-019, Sept. 1960.

"Stevens, F. L., On, T. J., and Clare, 'l. A., "Wrap-Around Vs. Cruciform Fins: Effects on Rocket Flight Performance," AIAA Paper 74-777, Aug. 1974.

${ }^{3}$ Lucero, E. F., "Subsonic Stability and Control. Characteristics of Configurations Incorporating Wrap-Around Surfaces," Journal of Spacecraft and Rockets, Vol. 13, Dec. 1976, pp. 740-745.
${ }^{4}$ Sawyer, W., Monta, W., Carter, W., and Alexander, W., "Control Characteristics for WrapAround Fins on Crutse Missile Configurations," AIAA Paper 81-0009, Jan. 1981.

${ }^{5}$ Daniels, P. and Hardy, S. R., "Roll-Rate Stabilization of a Misslle Configuration with Wrap-Around Fins," Journal of Spacecraft and Rockets, Vo1. 13, July 1976, pp. 446-448.

${ }^{6}$ Mermagen, W. H. and Oskay, V., "Yaw-sonde Tests of 2.75-Inch Mk 66 Mod I Rocket," U.S. $\Lambda$ rmy Armament Research and Developinent Command, Ballistic Research Laboratory, Aberdeen Proving Grounds, Maryland, ARBRL-MR-03127, Aug. 1981.

${ }^{7}$ Holmes, J. E., "Wrap-Around Fin (WAF) Aerodynamics," Proceedings, 9th Navy Symposium of Aeroballistics, Applied Physics Laboratories, the Johns Hopkins Untversity, Maryland, Paper No. 4, May 1972.

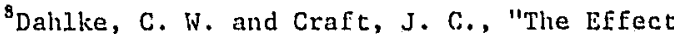
of Wrap-Around Fins on Aerodynamic Stability and Rolling Moment Variations," U.S. Army Missile Conmand, Redstone Arsenal, Alabama, RD-TR-73-1.7, July 1973.

${ }^{9}$ Dahike, C. W., "The Aerodynamic Characteristics of Wrap-Around Fins at Mach Numbers of 0.3 to 3.0," U.S. Army Missile Research, Development: and Engineering Laboratory, Redstone Arsenal, Alabama, $\mathrm{RD}-77-4$, OCt. 1976.

${ }^{10}$ Humphrey, J. A. and Dah1ke, C. W., "A Summary of Aerodynamic Characteristics of Wrap-Around Fins from Mach 0.3 to 3.0," U.S. Army Missile Research and Development Command, Redstone Arsenal, Alabama, TD-77-5, Nar. 1977.

${ }^{11}$ Rom, J., Zorea, C., and Gordon, R., "On the Calculation of Nonlinear Aerodynamic Characteristics and the Near Vortex Wake," ICAS Paper No. $74-27,1974$.

${ }^{22}$ Woodward, F. A., "An Improved Method for the Aerodynamic Analysis of Wing-Body-Tail ConEigurations in Subsonic and Supersonic Flow," NASA $\mathrm{CR}-2228$, May 1973.

13 Rusak, Z., Wasserstrom, E., and Seginer, $A$., "Numerical CaIculation of Nonlinear Aerodynamics of Wing-Body Configurations," AIAA Journal (Paper No. J13442), to be published July 1983.

14 von Kármán, T., "Calculation of Pressure Distribution on Airship Hulis," NACA TM 574, 1930.

${ }^{15}$ Goodwin, F. K. and Dillenius, M. F. E., "Extension of the Method for Predicting SixDegrees-of-Freedom Store Separation Irajectories at Speeds Up to the Critical Speed," Atr Force Fl.ight Dynamics Laboratory, Wright-patterson AFB, Ohio, AFFDL-TR-72-83, Oct. 1972.

${ }^{16}$ Bar-Haim, B., "Wrap-Around Fins Aerodynamics," M.Sc. Thesis, Technion-Israel Institute of Technology, Haifa, Israel, June 1981. 
${ }^{17}$ Rom, J., Victor, M., Reichenberg, M., and Salomon, M., "Wind Tunnel Measurements of the Base Pressure of an Axially Symmetric Model in Subsonic, Transonic and Supersonic Speeds at High Reynolds Numbers," Technion-Israel Institute of Technology, Haifa, Israel, TAE Rep. 134, July 1972.

$1{ }^{3}$ Merz, R. A., "Subsonic Axisymmetric Near Wake Studies," AIAA Journal, Vol. 16, July 1978, pp. 656-659.

${ }^{19}$ Little, B. H. and Whipkey, R. R., "Locked Vortex Afterbodies," Journal of Aircraft, Vol. 16, May 1979, pp. 296-302.

${ }^{20} \mathrm{Gai}$, S. L. and Patil, S. R., "Subsonic Axisymmetric Base Flow Experiments with Base Modifications," Journal of Spacecraft and Rockets, Vo1. 17, Jan. $-\mathrm{Feb}$. 1980, pp. 42-46.

${ }^{21}$ Weeks, T. M. and Allen, N., "Base Flow Studies in/and Calibration of the New 15-Inch AFFDL Transonic Facility," Air Force Flight Dynamics Laboratory, Wright-Patterson AFB, Ohio, Unpublished Data, May 1972 .

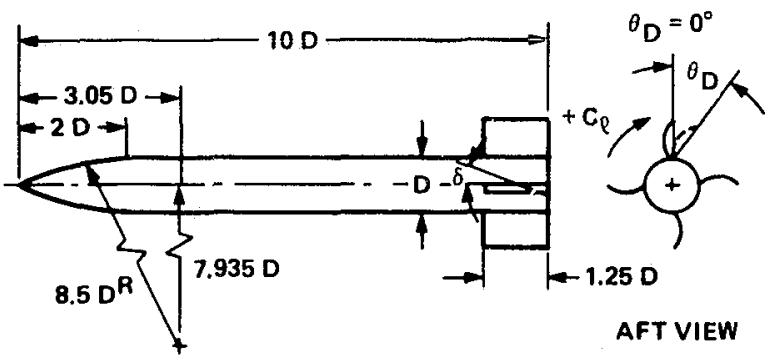

Fig. 1 Standard WAF configuration. ${ }^{7}$

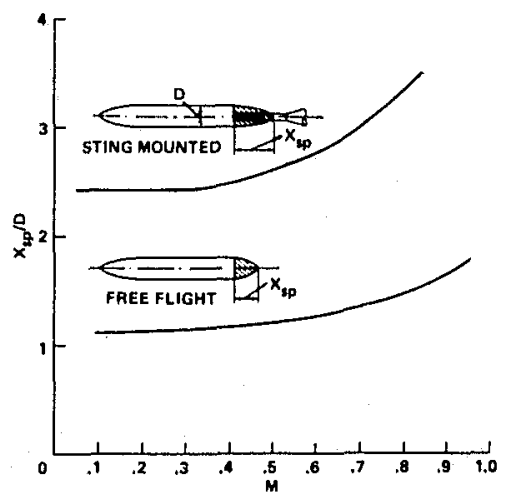

Fig. 2 Modeling of the closed wake in free fight and on a sting mount.
${ }^{22}$ Lee, G., "An Investigation of the Transonic Flow Fields Surrounding Hot and Cold Sonic Jets," NASA TN D-853, Apri1 1961.

${ }^{23}$ Love, E. S., Grigsby, C. E., Lee, L. P., and Woolding, M. J., "Experimental and Theoretical Studies of Axisymetric Free Jets," NASA TR R-6, 1959.

24 "Missile Engineering - Army MLRS Test-Launch at White Sands," Aviation Week and Space Technology, Vol. 112, No. 23, June 9, 1980, p. 52 .

${ }^{25}$ Bergbauer, D. M., Bergman, R. W., and Bentley, R., "Spin Profile Tailoring for the Improved 2.75" Rocket," AIAA Paper 80-1575, 1980.

\section{ORICINAL PRGE TS OF POOR QUALITY}

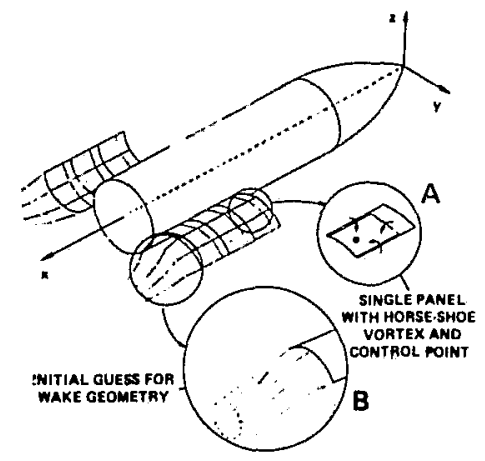

Fig. 3 Schematic modeling of WAF configurations, 16

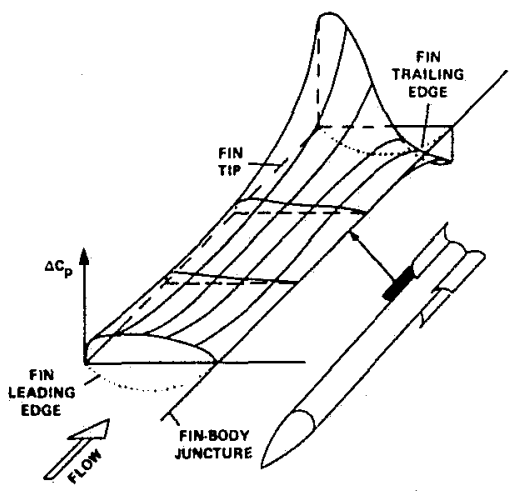

Fig. 4 Load distribution over fin on standard WAF configuration at $M=0$ and zero angle of attack. 


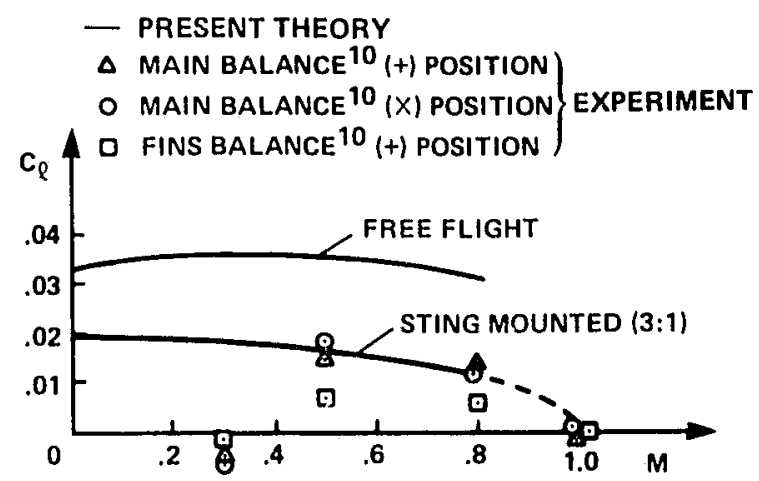

Fig. 5 Rolling-moment coefficient, compared with experimental daca (standard WAF configuration).

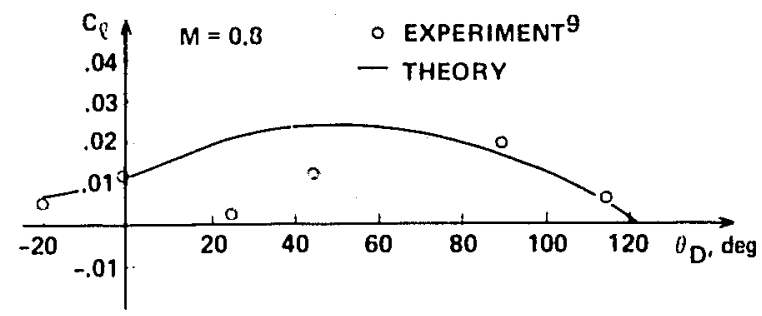

Fig. 6 Rolling-moment coefficient vs. Fin-opening angle compared with experimental data.

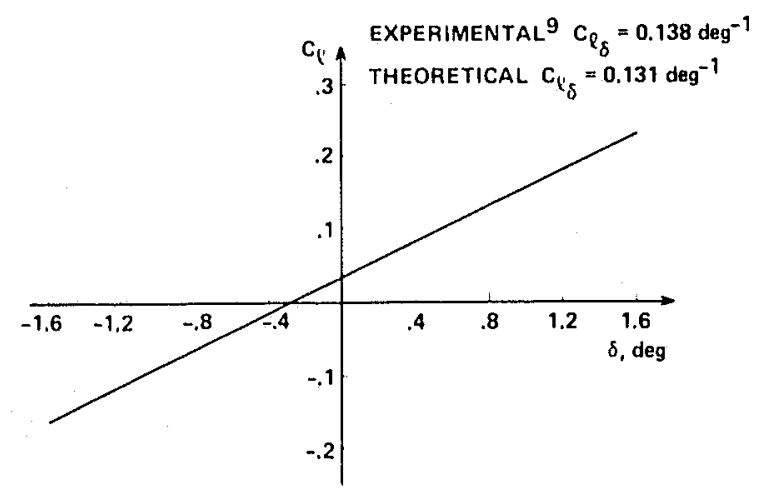

Fig. 7 Rolling-moment coefficients due to findifferential deflection.

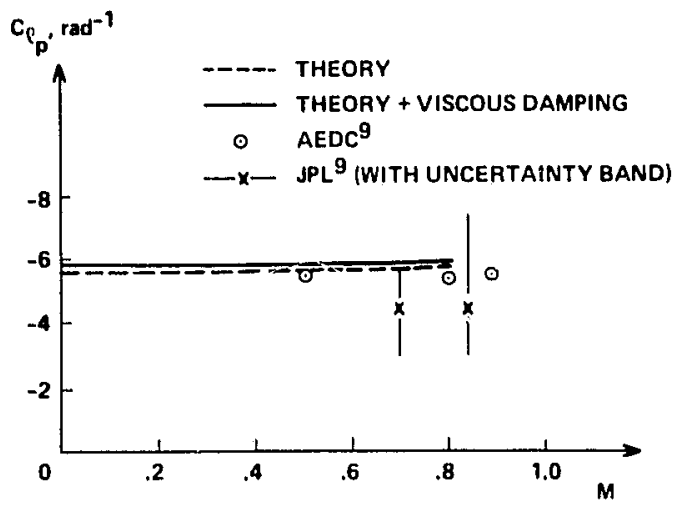

Fig. 8 Roll-damping-moment coefftcient, including body contribution, compared with the experimental data.

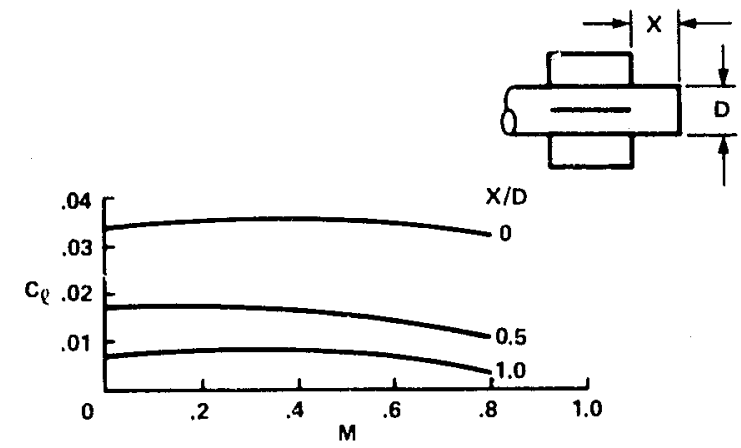

Fig. 9 Free-flight rolling-moment coefficient variation with fin axial position.

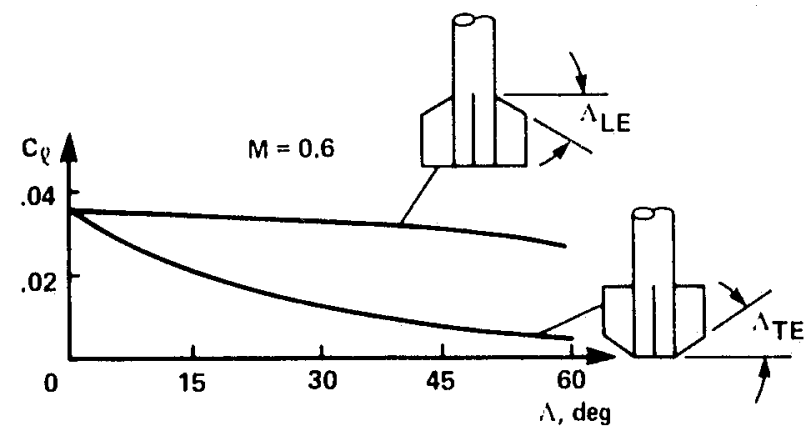

Fig. 1.0 Free-flight rolling-moment coefficient variation with leading-edge and trailing-edge sweep angle. 


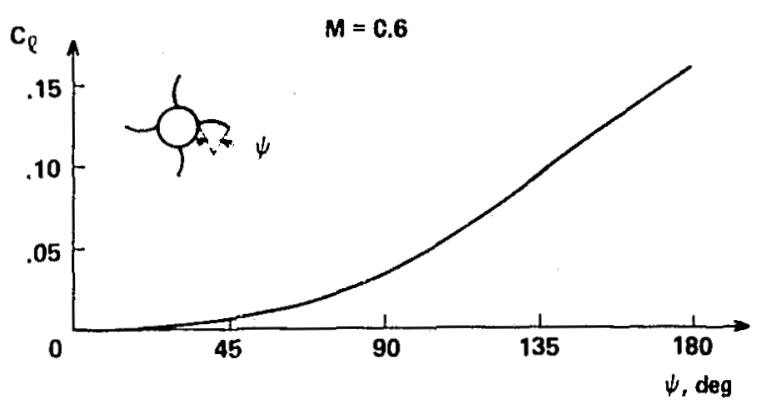

Fig. 11 Free-flight rolling-moment coefficient vs. fin span angle.

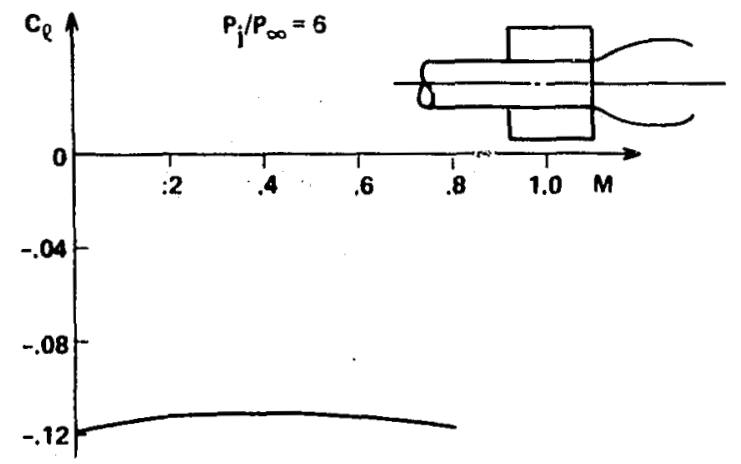

Fig. 12 Rolling-moment coefficient with a $6: 1$. pressure ratio jet plume.

ORIGIMAL PARE IS

OF POOR QUALITY 